\title{
Avaliação da intervenção de carreira: a perspectiva dos ex-clientes
}

\author{
Marina Noronba Ferraz de Arruda - Universidade de São Paulo, Ribeirão Preto, Brasil \\ Lucy Leal Melo-Silva 1 - Universidade de São Paulo, Ribeirão Preto, Brasil
}

\begin{abstract}
Resumo
A avaliação de estratégias de intervenções de carreira é considerada relevante na literatura internacional. Este estudo objetiva avaliar a intervenção de carreira realizada em um serviço-escola de um curso de psicologia de uma universidade pública brasileira. Participaram do estudo 77 ex-clientes jovens entre 17 e 24 anos, com predomínio de mulheres, e atendidos em grupo. O método de avaliação da intervenção é baseado no modelo de French, Hiebert e Bezanzon, que contem três dimensões de análise (input, processos e output). O questionário, enviado por correio, contém questões objetivas e dissertativas. Os resultados quantitativos foram analisados por meio de estatística descritiva e os dados qualitativos, por meio da análise de conteúdo. Em síntese, os ex-clientes avaliaram positivamente as condições oferecidas pelo serviço, valorizando as atividades e os temas trabalhados na intervenção. A incorporação de mais atividades informativas e a expansão do serviço foram sugeridas.

Palavras-chave: Orientação vocacional, Intervenção psicológica, Avaliação de programa, Avaliação de processo.
\end{abstract}

Evaluation of career intervention: former clients' perspectives

\begin{abstract}
Evaluation of career intervention strategies is considered relevant in international literature. This study aimed at evaluating career interventions carried out at a clinic-service delivered by a psychology course at a Brazilian state university. Seventyseven former clients, aged between 17-24 years old, with prevalence of females, and attended in groups, participated in this study. The intervention evaluation was based on French, Hiebert and Bezanzon's model, which contains three dimensions of analysis (input, processes and output). The questionnaire, forwarded by post, consisted of open and closed questions that contemplated three dimensions: inputs, processes and outcomes. The quantitative data were analyzed by means of descriptive statistics, and the qualitative data by means of content analysis. In summary, the former clients evaluated the service conditions positively and attributed positive value to the activities and themes developed in the interventions. The need for more career information and service expansion were suggested.

Keywords: Vocational guidance, Psychological intervention, Program evaluation Process assessment.
\end{abstract}

A intervenção em orientação profissional pode ser definida como "qualquer estratégia para ajudar um cliente a tomar e implementar decisões eficazes de carreira" (Spokane, 2004, p. 459). Objetivando verificar se a intervenção alcançou as metas, torna-se necessário avaliar. Por sua vez, a avaliação das intervenções em orientação profissional é caracterizada como uma forma de analisar práticas e instrumentos, com o objetivo de tornar o processo de intervenção mais eficaz e adequado a cada cenário e contexto. Para Walsh e Betz (1995), citados por Chartrand e Walsh (2001), o processo de avaliação de intervenções em desenvolvimento de carreira tem como objetivo colher informações para auxiliar as pessoas a compreenderem e a lidarem com problemas. Nesse sentido, Chartrand e Walsh (2001) complementam que, além de auxiliar a tomada de decisão, a avaliação contribuiria para melhorar o trabalho do orientador vocacional.

A avaliação da qualidade da intervenção pode ter como foco processos e resultados. O primeiro pode ser compreendido como a apreciação crítica dos

\footnotetext{
${ }^{1}$ Endereço para correspondência:

Av. Bandeirantes, 3.900 Bairro Monte Alegre - 14040-901 Ribeirão Preto-SP.

E-mail: lucileal@ffclrp.usp.br
}

componentes da intervenção, por meio de indicadores de qualidade que evidenciem a variação da eficácia de cada estratégia de intervenção. Já o segundo propõe estimar o impacto da intervenção em função dos progressos do cliente após o término do processo. Considera-se, neste estudo, relevante avaliar tanto os instrumentos utilizados na prática, quanto os processos e resultados da intervenção em orientação profissional (Melo-Silva \& Jacquemin, 2001), sendo essencial promover o intercâmbio entre esses fatores, relacionando "como" e "quais" mudanças ocorreram, para um maior aproveitamento da avaliação (Swanson, 1995).

A literatura estrangeira destaca que a importância da realização da avaliação das práticas de orientação profissional se dá por evidenciar quais aspectos encontram-se satisfatórios (Brown, 2003), quais conhecimentos e competências precisam ser desenvolvidas e quais atitudes se devem adotar (Guichard \& Huteau, 2001). A avaliação de processos e resultados contribui, ainda, para o planejamento da intervenção ante os objetivos propostos (Melo-Silva \& Jacquemin, 2001), sendo essencial considerar os objetivos e expectativas dos clientes no que tange aos resultados a serem alcançados (Amundson, HarrisBowlsbey \& Niles, 2005). 
Vale ressaltar que a compreensão acerca das aplicações e efeitos, vantagens e limites do processo de orientação profissional, advinda da avaliação da intervenção, influencia a eficiência da intervenção, acarreta em uma diminuição dos gastos e possibilita uma melhoria na assistência oferecida aos clientes (Spokane, 2004). Afinal, a simples reflexão sobre o processo e os procedimentos nele utilizados promove o enriquecimento do serviço avaliado (Whiston \& Buck, 2008). Dessa forma, pelo estudo de processos e resultados de intervenções em orientação profissional, pode-se demonstrar não só seus efeitos sobre a tomada de decisão e desenvolvimento de carreira, mas, ainda, quais as formas mais satisfatórias dessa intervenção.

O questionamento sobre a eficácia das intervenções de carreira é recorrente, sobretudo nos Estados Unidos, desde a década de 1940. Para Spokane (2004, p. 462), o número de estudos "continua a aumentar, ainda que de forma lenta". Mais recentemente, destacam-se os estudos de meta-análise de Brown e Ryan Kane (2000) e Whiston, Brecheisen e Stephens (2003), que tiveram como foco, respectivamente, relação entre variáveis do processo e a escolha profissional e comparação da eficácia entre modalidades de intervenção.

No Brasil, pesquisas de avaliação de resultados e processos são, ainda, pouco frequentes. Destacam-se investigações de caracterização dos serviços e clientela, resolutividade do atendimento; descrição de técnicas empregadas, a avaliação da intervenção em função da maturidade para escolha dos clientes, a formação e a identidade profissional do psicólogo e/ou do orientador. A avaliação de processos e resultados em estudos mais aprofundados tem sido realizada mais recentemente (Almeida, 2003; Almeida \& Melo-Silva, 2006; Arruda, 2009; Fraga, 2003; Lassance, Bardagi \& Teixeira, 2009; Loosli, 2003; Melo-Silva \& Jacquemin, 2001; Yokoyama, 2009).

A despeito dos estudos mencionados anteriormente, a literatura traz de forma recorrente que poucos trabalhos foram realizados para avaliar intervenções de carreira. Estudos canadenses apontam para uma valorização da prática avaliativa por parte dos orientadores profissionais. No entanto, a implementação das avaliações junto aos clientes continua pouco frequente (Lalande, Hiebert, Magnusson, Bezanson \& Borgen, 2006; Lalande \& Magnusson, 2007 citados por Baudouin e colaboradores, 2007). Quando a avaliação é realizada e sistematizada, Conger, Hiebert e HongFartell (1993), citados por Hiebert (1994) destacam que vários problemas são encontrados. Um deles consiste na falta de cientificidade e fidedignidade de pesquisas feitas informalmente pelos próprios orientadores vocacionais, durante a intervenção ou ao término da sessão. Outro problema apontado refere-se à priorização de outras atividades em detrimento da avaliação dos serviços prestados, ainda, ao curto período destinado para o processo de orientação profissional. E, por fim, o receio do profissional de obter resultados negativos e, assim, "falhar" em seu teste de qualidade, ignorando as benesses que a prática avaliativa oferece quando bem realizada.

Considerando a necessidade de avaliação de resultados e processos e de realização de novos estudos acerca dessa temática, o presente estudo dá continuidade à linha investigativa sobre avaliação da intervenção em orientação profissional desenvolvida no Serviço de orientação profissional (SOP) da Faculdade de Filosofia, Ciências e Letras de Ribeirão Preto da Universidade de São Paulo (FFCLRP/USP), tendo como objetivo avaliar a intervenção em orientação profissional sob a perspectiva dos exclientes atendidos (Arruda, 2009).

\section{Método}

\section{Participantes}

No período investigado, entre 2001 e 2006, somaram-se 1.207 inscritos no serviço, que compuseram a população potencial. Após contato telefônico, 536 se dispuseram a responder ao questionário, que, então, lhes foi enviado. A amostra final foi constituída de 77 participantes, sendo 61 concluintes, 11 abandonadores (iniciaram o processo e interromperam antes do término programado) e cinco (5) desistentes (fizeram a inscrição, porém não iniciaram o atendimento). A amostra foi composta por 62 mulheres $(80,5 \%)$ e 15 homens $(19,5 \%)$, atendidos individualmente $(\mathrm{n}=21 ; 27,3 \%)$ ou em grupo $(\mathrm{n}=56$; 72,7). Os participantes são majoritariamente provenientes da cidade sede da universidade do interior do estado de São Paulo, Brasil ( $\mathrm{n}=68 ; 88,3 \%)$ e de cidades da região ( $\mathrm{n}=9 ; 11,7 \%)$. Apresentavam, no momento da pesquisa, idades compreendidas entre 17 e 54 anos (médiaidade $=24 ; \mathrm{DP}_{\text {idade }}=9,03$ ) e, em sua totalidade, possuíam como escolaridade o Ensino Médio completo ( $\mathrm{n}=77,100 \%)$.

\section{Instrumentos}

A literatura aponta vantagens e desvantagens para a utilização de questionários em pesquisa, no processo de coleta de dados (Selltiz, Wrightsman \& Cook, 1987). Apesar da baixa porcentagem de retorno dos questionários, optou-se por utilizar tal ferramenta na coleta de dados, principalmente pela necessidade de alcance geográfico e pelo grande número de ex-clientes 
que passaram pelo Serviço de Orientação Profissional (SOP) no período delimitado para o estudo $(n=1207)$.

Definiu-se pela utilização de versões do Questionário de Avaliação de Orientação Profissional (QAOP) de Almeida (2003) e Almeida e Melo-Silva (2006). O QAOP contém questões objetivas do tipo Likert de 5 pontos e dissertativas. As versões do QAOP foram construídas seguindo o sistema proposto por French, Hiebert e Bezanzon (1994) e contemplam, de forma adaptada à realidade do SOP, três dimensões de análise, a saber: (1) condições oferecidas pelo serviço (localização, sala de atendimento, inscrição no serviço, entrevista de triagem, duração dos atendimentos e do processo, recursos materiais e humanos disponíveis); (2) processos (atividades desenvolvidas, interação com o estagiário e demais participantes, atividades complementares, assiduidade, grau de importância conferido aos temas trabalhados), e (3) resultados (maturidade para a tomada de decisão profissional, influências percebidas nessa escolha, auxílio oferecido pela orientação profissional na resolução de conflitos e desenvolvimento de habilidades necessárias para a organização de um projeto de vida profissional). É importante ressaltar que, no caso dos ex-clientes desistentes, o questionário contém apenas a primeira e a última dimensão de análise (respectivamente: inputs e resultados) uma vez que não participaram do processo de orientação profissional.

\section{Procedimento de coleta de dados}

Em 2007, foi feito o levantamento de todos os clientes triados no período delimitado para estudo, definidos como a população inicial $(n=1.207)$, e realizados contatos telefônicos com esses ex-clientes, a fim de reduzir o envio desnecessário de material, motivar a participação e ampliar o comprometimento com o estudo por parte dos ex-clientes voluntários, que concordaram, a priori, em colaborar com o projeto e compuseram a população potencial $(n=536)$.

Os materiais para coleta de dados (um envelope contendo o QAOP, a carta explicativa, o Termo de Consentimento Livre e Esclarecido e um envelope-resposta subscrito e pré-franqueado) foram organizados e enviados pelo correio para a população potencial, no final de 2007. Após o prazo de um mês, foram reestabelecidos contatos telefônicos com os exclientes que, em princípio, concordaram em participar da pesquisa, mas que, até o momento, não haviam respondido, visando confirmar o recebimento e estimular a resposta aos questionários. Em alguns casos, os impressos foram reenviados. Ao final do período estipulado para o recebimento dos questionários, somaram-se 77 ex-clientes respondentes, que compuseram a amostra final do estudo.

No que se refere aos aspectos éticos, cabe ressaltar que o projeto foi aprovado pelo Comitê de Ética em Pesquisa da Universidade e que foram tomados os devidos cuidados relativos à fidelidade das fontes informativas e aos princípios de benevolência e não-maleficência. Considerando que a pesquisa abordou a questão da orientação profissional desenvolvida em um momento de tomada de decisão relativa à carreira, os pesquisadores se colocaram à disposição dos participantes para realizar entrevistas. No entanto, não houve solicitação para atendimento.

\section{Procedimento de análise dos dados}

Os resultados obtidos nas questões objetivas foram tratados quantitativamente, por meio de estatística descritiva, e as informações obtidas nos comentários e questões dissertativas foram tratadas qualitativamente, utilizando a análise de conteúdo proposta por Bardin (1979). Neste estudo, tal sistematização seguiu as seguintes etapas: (1) préanálise: organização e sistematização das idéias iniciais, por meio de uma leitura flutuante dos comentários realizados pelos ex-clientes nos espaços para livre manifestação dos questionários e das respostas às questões abertas; (2) codificação: organização dos dados em unidades de registro (núcleos de sentido) e agrupamento conforme a similaridade de conteúdo, visando a uma categorização em temas de análise; e (3) inferência referencial: discussão dos dados, considerando os indicadores presentes no material analisado e a situação de comunicação, sob a perspectiva da orientação profissional.

\section{Resultados e Discussão}

A primeira parte do questionário apresenta 11 itens para a avaliação das condições oferecidas pelo SOP/CPA/FFCLRP/USP (Tabela 1), respondidas de acordo com uma escala Likert de 5 pontos (péssimo, ruim, regular, bom ou excelente).

Em 10 dos 11 itens avaliados, predominou o conceito "bom", entre $40 \%$ a $50 \%$ das respostas, exceto no item referente ao tempo da sessão individual, no qual a maioria deixou a questão sem resposta, em decorrência da predominância de atendimento em grupo. A localização da USP (no campus universitário) obteve uma avaliação positiva. Nos comentários livres, alguns participantes julgam a localização favorável, enquanto outros apontam a dificuldade encontrada em relação a ela, como mostram os registros a seguir: "a localização era muito boa [...]" (mulher, 20 anos, atendida em grupo, em 
Tabela 1 - Distribuição das respostas dos participantes $(n=77)$ por item, em função do conceito atribuído às condições oferecidas

\begin{tabular}{|c|c|c|c|c|c|c|}
\hline Itens & Péssimo & Ruim & Regular & Bom & Excelente & Sem resposta \\
\hline & (f) $\%$ & (f) $\%$ & (f) $\%$ & (f) $\%$ & (f) $\%$ & (f) $\%$ \\
\hline 1. Localização da USP & (0) 0 & (8) 10,4 & (28) 36,4 & (31) 40,3 & (9) 11,7 & (1) 1,3 \\
\hline 2. Sala de Atendimento & (0) 0 & (4) 5,2 & (18) 23,4 & (45) 58,4 & (6) 7,8 & (4) 5,2 \\
\hline 3. Inscrição no serviço & (0) 0 & (0) 0 & (13) 16,9 & (51) 66,2 & (10) 13,0 & (3) 3,9 \\
\hline $\begin{array}{l}\text { 4. Triagem } \\
\text { 5. Tempo da sessão }\end{array}$ & (0) 0 & (0) 0 & (9) 11,7 & (46) 59,7 & (12) 15,6 & (10) 13,0 \\
\hline $\begin{array}{l}\text { individual }(50 \mathrm{~min}) \\
\text { 6. Tempo do encontro }\end{array}$ & (1) 1,3 & (0) 0 & (3) 3,9 & (22) 28,6 & (18) 23,4 & (33) 42,9 \\
\hline $\begin{array}{l}\text { em grupo ( } 2 \text { horas }) \\
\text { 7. O tempo de duração }\end{array}$ & (0) 0 & (0) 0 & (3) 3,9 & (39) 50,6 & (15) 19,5 & (20) 26,0 \\
\hline do processo (sessões) & (0) 0 & (0) 0 & (9) 11,7 & (43) 55,8 & (15) 19,5 & (10) 13,0 \\
\hline disponíveis & (0) 0 & (3) 3,9 & (15) 19,5 & (35) 45,5 & (16) 20,8 & (8) 10,4 \\
\hline $\begin{array}{l}\text { 9. Competência do } \\
\text { estagiário }\end{array}$ & (0) 0 & (4) 5,2 & (8) 10,4 & (33) 42,9 & (28) 36,4 & (4) 5,2 \\
\hline $\begin{array}{l}\text { 10. Atendimento da } \\
\text { secretatia (Casa } 7 \text { e } 34)\end{array}$ & (0) 0 & (0) 0 & (4) 5,2 & (40) 51,9 & (15) 19,5 & (18) 23,4 \\
\hline secretaria $(\mathrm{CPA})$ & (0) 0 & (0) 0 & (3) 3,9 & (34) 44,2 & (10) 13,0 & (30) 39,0 \\
\hline
\end{tabular}

2004)2; " a USP fica localizada muito distante da minha casa [...]" (mulher, 19 anos, atendida em grupo, em 2005).

As condições das salas de atendimento obtiveram $66,2 \%$ de opiniões favoráveis, somando os conceitos "bom" (58,4\%) e "excelente" (7,8\%), porém nos espaços para livre manifestação dos participantes foram feitas algumas críticas sobre as condições das salas de atendimentos e seus efeitos para o processo de orientação profissional. Os registros foram predominantemente referentes a um dos locais onde os atendimentos ocorriam até 2004, que apresentava uma limitação em termos de infraestrutura, sendo este o motivo do item fazer parte do questionário e um dos argumentos da equipe para a mudança do local. Uma participante registrou: "o atendimento na Casa 7 julgo como desfavorável ao andamento de todo o processo, pois a casinha era muito pequena para o grupo, abafada, limitando as atividades" (mulher, 19 anos, atendida em grupo, em 2004).

Em seguida, o foco da avaliação se dirigiu ao processo de orientação profissional, tanto no que se refere às estratégias utilizadas (Tabela 2), como aos temas trabalhados (Tabela 3). Nas duas partes, solicitou-se a atribuição de um conceito para avaliação de itens que contribuíram para o processo da tomada de decisão, segundo uma escala Likert de 5 pontos.

\footnotetext{
2 Foram omitidos os nomes dos participantes, como forma de garantir o sigilo de suas identidades. São referidos o sexo, a idade, a modalidade de atendimento e o ano de atendimento.
}

Em uma visão geral, os dados apontam para uma avaliação positiva das estratégias e dos temas desenvolvidos no processo de orientação profissional. No espaço destinado aos comentários, a maioria dos participantes explicitou o auxílio da orientação profissional em sua escolha, além de abordarem a estrutura das sessões, a importância da informação profissional e o papel dos estagiários no processo.

O item 1, as atividades facilitaram a decisão obteve avaliação geral positiva, porém com valor elevado do conceito "muito pouco", merecendo maior atenção. Nos comentários, os participantes destacam contribuições para a tomada de decisão:

$$
\begin{aligned}
& \text { a metodologia utilizada nas minhas sessões em grupo } \\
& \text { foi muito importante para minha decisão, fazendo } \\
& \text { descobrir mais sobre os cursos e sobre os meus gostos. O } \\
& \text { método de relacionar fotos, jogos, teatro, música, } \\
& \text { fábulas, desenho e debates sobre a decisão e os cursos } \\
& \text { foi enriquecedor [...] (mulher, } 20 \text { anos, atendida } \\
& \text { em grupo, em 2004) }
\end{aligned}
$$

No entanto, foram registrados questionamentos quanto a sua validade para ajudar na escolha profissional, provavelmente pela expectativa de uma posição mais ativa do orientador profissional, presentes tanto nos comentários, como na questão dissertativa que abordava as sugestões dos participantes, tais como, "direcionar mais o aluno, e encaminhá-lo para estágio, na área pré estabelecida, e depois, o reavaliar se está na área mais próxima, ou até 
Tabela 2 - Distribuição das respostas dos participantes ( $\mathrm{n}=77)$ em função dos conceitos atribuídos aos itens que contribuíram para a tomada de decisão

\begin{tabular}{|c|c|c|c|c|c|c|}
\hline \multirow[t]{2}{*}{ Itens } & $\begin{array}{l}\text { Muito } \\
\text { pouco }\end{array}$ & Pouco & $\begin{array}{c}\text { Mais ou } \\
\text { menos }\end{array}$ & Muito & Totalmente & Sem resposta \\
\hline & (f) $\%$ & (f) $\%$ & (f) $\%$ & (f) $\%$ & (f) $\%$ & (f) $\%$ \\
\hline 1. As atividades facilitaram a decisão & (7) 9,1 & (4) 5,2 & (19) 24,7 & (33) 42,9 & (5) 6,5 & (9) 11,7 \\
\hline $\begin{array}{l}\text { 2. } \mathrm{O} \text { vínculo com o estagiário me } \\
\text { ajudou } \\
\text { 3. } \mathrm{O} \text { vínculo com os integrantes do }\end{array}$ & (6) 7,8 & (9) 11,7 & (20) 26,0 & (22) 28,6 & (15) 19,5 & (5) 6,5 \\
\hline $\begin{array}{l}\text { grupo me ajudou } \\
\text { 4. Minha participação contribuiu }\end{array}$ & (4) 5,2 & (5) 6,5 & (21) 27,3 & (22) 28,6 & (7) 9,1 & (18) 23,4 \\
\hline para o processo & (2) 2,6 & (1) 1,3 & (25) 32,5 & (25) 32,5 & (9) 11,7 & (15) 19,5 \\
\hline 5. Realizei as atividades & & & & & & \\
\hline complementares (externamente) & (12) 15,6 & (7) 9,1 & (13) 16,9 & (14) 18,2 & (9) 11,7 & (22) 28,6 \\
\hline 6. Fui assíduo às sessões & (3) 3,9 & (2) 2,6 & (7) 9,1 & (16) 20,8 & (41) 53,2 & (8) 10,4 \\
\hline $\begin{array}{l}\text { 7. Tomei consciência sobre o meu } \\
\text { processo de escolha } \\
\text { 8. O teste de fotos contribuiu para o }\end{array}$ & (5) 6,5 & (2) 2,6 & (7) 9,1 & (29) 37,7 & (22) 28,6 & (12) 15,6 \\
\hline processo de escolha & (3) 3,9 & (3) 3,9 & (27) 35,1 & (15) 19,5 & (14) 18,2 & (15) 19,5 \\
\hline
\end{tabular}

a profissão ideal a suas abilidades [sic]" (homem, 39 anos, atendido em grupo, em 2003)3. "A orientação profissional deveria ser mais incisiva, mais objetiva. Testes de aptidão seriam aplicados" (homem, 53 anos, desistente em 2002).

Nesse sentido, os ex-clientes, atendidos e até um desistente, como no caso anterior, parecem ainda esperar intervenção diretiva, na qual o orientador profissional assuma um papel mais ativo, por exemplo, na aplicação de testes, visando o ajuste entre os interesses e aptidões como possibilidades de escolha, conforme modelo traço e fator. A intervenção realizada se baseia na modalidade clínica, proposta por Bohoslavsky (2003), na qual o psicólogo assume uma postura não-diretiva, oferecendo informações e esclarecimentos com o objetivo de favorecer uma decisão autônoma por parte do indivíduo, que assume papel ativo e é tido como capaz de enfrentar e resolver a situação conflituosa, o que muitas vezes os clientes só compreendem ao término do atendimento. Encontrar o ponto de equilíbrio, entre uma intervenção menos diretiva e as expectativas do orientando de ter "soluções mágicas", constitui um grande desafio para os profissionais.

Quanto à realização de atividades complementares (item 5) obteve-se o maior índice de questões sem resposta no conjunto dos itens da Tabela 2. Verifica-se grande dispersão nas respostas entre os conceitos, indicando que para alguns clientes a busca de informações fora dos atendimentos (em guias, internet e com profissionais da área de atuação

\footnotetext{
${ }^{3}$ Foram mantidas as grafias dos ex-clientes nas respostas por escrito às questões dissertativas do QAOP.
}

pretendida), proposta pelos psicólogos estagiários, foi realizada satisfatoriamente, auxiliando em seu desenvolvimento de carreira. Para muitos não foi uma atividade priorizada. Pode-se inferir que isso se deva a inúmeros motivos, por exemplo, falta de comprometimento com a tarefa de escolha e/ou por outras questões de ordem pessoal do cliente. Também pode ter sido por ausência de planejamento de uso do tempo, recursos materiais ou de oportunidades de contato com profissionais da área de interesse e por outras questões contextuais. E, ainda, falta de motivação para realização das atividades extraatendimento. No que se refere ao serviço, torna-se importante buscar novas estratégias, visando ampliar a frequência de realização dessas atividades, consideradas essenciais para a exploração das possibilidades, maturação da escolha e sucesso do processo de orientação profissional.

A segunda parte da avaliação dos processos se referiu aos temas trabalhados (Tabela 3), avaliados positivamente e considerados pertinentes.

Os itens escolha da carreira (1) e informações sobre as profissões (7) receberam os maiores índices de avaliações "muita" e "extrema", o que mostra grande importância atribuída, talvez por se constituírem os principais eixos da orientação profissional, tanto no que diz respeito à busca pelo atendimento, quanto à intervenção propriamente dita. Observa-se que a maioria dos ex-clientes considera essencial a busca e o recebimento de informações sobre as carreiras. Dessa forma, tais temas são fundamentais no que se refere às competências requeridas do orientador profissional. 
Tabela 3 - Distribuição das respostas dos participantes $(n=77)$ em função do conceito atribuído à importância dos temas trabalhados

\begin{tabular}{|c|c|c|c|c|c|c|}
\hline Itens & Nenhuma & Pouca & Média & Muita & Extrema & Sem resposta \\
\hline & (f) $\%$ & (f) $\%$ & (f) $\%$ & (f) $\%$ & (f) $\%$ & (f) $\%$ \\
\hline 1. Escolha da carreira & (3) 3,9 & (2) 2,6 & (6) 7,8 & (26) 33,8 & (31) 40,3 & (9) 11,7 \\
\hline 2. Medo do vestibular & (3) 3,9 & (8) 10,4 & (12) 15,6 & (25) 32,5 & (16) 20,8 & (13) 16,9 \\
\hline 3. Medo de errar & (3) 3,9 & (5) 6,5 & (9) 11,7 & (28) 36,4 & (19) 24,7 & (13) 16,9 \\
\hline 4. Influência da família & (9) 11,7 & (9) 11,7 & (15) 19,5 & (21) 27,3 & (12) 15,6 & (11) 14,3 \\
\hline 5. Autoconhecimento & (0) 0 & (2) 2,6 & (12) 15,6 & (32) 41,6 & (20) 26,0 & (11) 14,3 \\
\hline 6. Medo do mundo adulto & (8) 10,4 & (20) 26,0 & (15) 19,5 & (14) 18,2 & (5) 6,5 & (15) 19,5 \\
\hline $\begin{array}{l}\text { 7. Informação sobre as } \\
\text { profissões }\end{array}$ & (2) 2,6 & (2) 2,6 & (10) 13,0 & (22) 28,6 & (30) 39,0 & (11) 14,3 \\
\hline 8. Mercado de trabalho & (1) 1,3 & (4) 5,2 & (13) 16,9 & (22) 28,6 & (26) 33,8 & (11) 14,3 \\
\hline 9. Transformações no & & & & & & \\
\hline mundo do trabalho & (1) 1,3 & (3) 3,9 & (17) 22,1 & (30) 39,0 & (13) 16,9 & (13) 16,9 \\
\hline 10. Dedicação aos estudos & (1) 1,3 & (2) 2,6 & (21) 27,3 & (23) 29,9 & (16) 20,8 & (14) 18,2 \\
\hline 11. Escola pública e privada & (2) 2,6 & (7) 9,1 & (17) 22,1 & (25) 32,5 & (11) 14,3 & (15) 19,5 \\
\hline $\begin{array}{l}\text { 12. Amizade entre os } \\
\text { participantes }\end{array}$ & (2) 2,6 & (11) 14,3 & (18) 23,4 & (17) 22,1 & (13) 16,9 & (16) 20,8 \\
\hline
\end{tabular}

Outro tema que pode ser destacado discorre sobre a influência da família (item 4), que também mostra uma certa distribuição entre os conceitos, ainda que predomine o conceito "muito" (27\%). Possivelmente, isso esteja relacionado à variação subjetiva quanto à percepção e ao grau de importância que o ex-cliente confere à influência familiar. Nos espaços de livre manifestação, os participantes valorizam tal discussão, mas dificilmente relatam ter recebido a influência de familiares, sendo frequente $o$ apontamento da ocorrência em outras famílias, o que reafirma a importância do tema estar presente no planejamento da intervenção. Alguns registros são transcritos a seguir: "Trabalhar sobre a influência da família é muito importante pois muitos pais 'obrigam' o filho a escolher determinada carreira" (mulher, 21 anos, atendida em grupo, em 2004).

O que mais me marcou durante os encontros foi sobre "medo de errar" e "influência da familia", onde pude tirar conclusões que nunca podemos ter medo de errar e que a decisão profissional é nossa e não da familia. (homem, 19 anos, atendido em grupo, em 2006)

No meu caso não tinha problemas com a família, eles até me apoiavam, mas nos encontros e conversas ficou evidente que ainda tem muitos pais que pressionam $e$ decidem o curso para os filhos. (mulher, 20 anos, atendida em grupo, em 2004)

Houve uma desistente que usou um dos espaços para comentários para falar sobre a influência recebida e seus efeitos para sua vida profissional: “mesmo sem saber o perfil 'certo', quis fazer moda ou arquitetura. Meu pai não queria que eu fizesse arquitetura e minha mãe queria moda, porque ela gosta [...]" (mulher, 22 anos, desistente, em 2002). Cabe lembrar que os questionários dirigidos aos desistentes não continham a parte de itens sobre o processo, uma vez que não participaram da intervenção. Ainda assim emerge a questão das influências nas respostas. A discussão sobre este item - influência da família - se mostra relevante no processo de escolha profissional.

Quanto aos resultados da intervenção de carreira, como mostra a Tabela 4, os participantes reconhecem o papel da orientação profissional para o amadurecimento de sua escolha, assim como a eficiência da intervenção para outras pessoas.

Além disso, parece que alguns participantes não consideram as influências recebidas da família, amigos, professores, mercado de trabalho e fatores socioeconômicos como relevantes para suas escolhas. Por sua vez, registram mais influências dos fatores educacionais e da própria intervenção realizada em suas decisões de carreira, como aponta a participante a seguir: "Minha escolha foi pouco influenciada por outras pessoas, depois do curso de Orientação Profissional tive certeza de qual carreira seguir" (mulher, 19 anos, atendida em grupo, em 2005).

Por fim, demonstraram satisfação quanto ao desenvolvimento de habilidades como o estabelecimento de objetivos realísticos, o reconhecimento de seus valores, o desenvolvimento de habilidades para trabalhar em equipe, organizar um projeto de vida e tomar a decisão profissional; realizado (ou ao menos iniciado) durante a intervenção em orientação profissional. 
Tabela 4 - Distribuição das respostas dos participantes ( $\mathrm{n}=77)$ em função do conceito atribuído aos itens

\begin{tabular}{|c|c|c|c|c|c|c|}
\hline Item & Nada & Pouco & $\begin{array}{c}\text { Mais ou } \\
\text { menos }\end{array}$ & Muito & $\begin{array}{l}\text { Total- } \\
\text { mente }\end{array}$ & Sem resposta \\
\hline 1- Acredito que: & & & & & & \\
\hline $\begin{array}{l}\text { Amadureci em relação à minha } \\
\text { decisão profissional } \\
\text { A OP foi fundamental para minha } \\
\text { decisão } \\
\text { O tipo de OP realizada pode ajudar } \\
\text { outras pessoas }\end{array}$ & $\begin{array}{l}\text { (4) } 5,2 \\
\text { (4) } 5,2 \\
\text { (1) } 1,3\end{array}$ & $\begin{array}{l}\text { (10) } 13,0 \\
\text { (14) } 18,2 \\
\text { (1) } 1,3\end{array}$ & $\begin{array}{l}\text { (16) } 20,8 \\
\text { (23) } 29,9 \\
\text { (9) } 11,7\end{array}$ & $\begin{array}{l}\text { (29) } 37,7 \\
\text { (19) } 24,7 \\
\text { (37) } 48,1\end{array}$ & $\begin{array}{l}\text { (17) } 22,1 \\
\text { (10) } 13,0 \\
\text { (26) } 33,8\end{array}$ & $\begin{array}{l}\text { (1) } 1,3 \\
\text { (7) } 9,1 \\
\text { (3) } 3,9\end{array}$ \\
\hline 2. Escolha da carreira foi influenciada: & & & & & & \\
\hline Pela família & (40) 51,9 & (13) 16,9 & (9) 11,7 & (7) 9,1 & (2) 2,6 & (6) 7,8 \\
\hline Pelos amigos & (41) 53,5 & (19) 24,7 & (8) 10,4 & (3) 3,9 & (1) 1,3 & (5) 6,5 \\
\hline Pelos meus professores & (40) 51,9 & (10) 13,0 & (10) 13,0 & (8) 10,4 & (2) 2,6 & (7) 9,1 \\
\hline Pelo mercado de trabalho & (23) 29,9 & (10) 13,0 & (14) 18,2 & (18) 23,4 & (6) 7,8 & (6) 7,8 \\
\hline Por fatores socioeconômicos & (20) 26,0 & (16) 20,8 & (13) 16,9 & (14) 18,2 & (7) 9,1 & (7) 9,1 \\
\hline Por fatores educacionais e sociais & (9) 11,7 & (12) 15,6 & (19) 24,7 & (20) 26,0 & (9) 11,7 & (8) 10,4 \\
\hline Pela orientação profissional & (12) 15,6 & (16) 20,8 & (20) 26,0 & (18) 23,4 & (6) 7,8 & (5) 6,5 \\
\hline $\begin{array}{l}\text { Pelos meios de comunicação: } \\
\text { televisão, revistas, internet }\end{array}$ & (19) 24,7 & (17) 22,1 & (19) 24,7 & (14) 18,2 & (3) 3,9 & (5) 6,5 \\
\hline $\begin{array}{l}\text { 3. A orientação profissional auxiliou-me } \\
\text { a: }\end{array}$ & & & & & & \\
\hline $\begin{array}{l}\text { Lidar com dificuldades } \\
\text { socioeconômicas } \\
\text { Lidar com conflitos pessoais e } \\
\text { familiares } \\
\text { Lidar com dificuldades educacionais }\end{array}$ & $\begin{array}{l}\text { (15) } 19,5 \\
\text { (16) } 20,8\end{array}$ & $\begin{array}{l}\text { (22) } 28,6 \\
\text { (15) } 19,5\end{array}$ & $\begin{array}{l}\text { (18) } 23,4 \\
\text { (20) } 26,0\end{array}$ & $\begin{array}{l}\text { (11) } 14,3 \\
\text { (17) } 22,1\end{array}$ & $\begin{array}{l}\text { (1) } 1,3 \\
\text { (7) } 9,1 \\
\text { (4) } 5,2\end{array}$ & $\begin{array}{l}\text { (8) } 10,4 \\
\text { (4) } 5,2 \\
\text { (5) } 6,5\end{array}$ \\
\hline Estabelecer objetivos realísticos & (5) 6,5 & (7) 9,1 & (20) 26,0 & (33) 42,9 & (7) 9,1 & (5) 6,5 \\
\hline Reconhecer meus valores & (2) 2,6 & (5) 6,5 & (20) 26,0 & (34) 44,2 & (11) 14,3 & (5) 6,5 \\
\hline $\begin{array}{l}\text { Desenvolver habilidades de } \\
\text { liderança } \\
\text { Desenvolver habilidades para }\end{array}$ & (7) 9,1 & (15) 19,5 & (22) 28,6 & (22) 28,6 & (7) 9,1 & (4) 5,2 \\
\hline $\begin{array}{l}\text { trabalhar em equipe } \\
\text { Desenvolver habilidades para }\end{array}$ & (3) 3,9 & (7) 9,1 & (25) 32,5 & (29) 37,7 & (7) 9,1 & (6) 7,8 \\
\hline $\begin{array}{l}\text { resolver problemas } \\
\text { Desenvolver habilidades para }\end{array}$ & (3) 3,9 & (12) 15,6 & (26) 33,8 & (26) 33,8 & (9) 11,7 & (1) 1,3 \\
\hline organizar um projeto de vida & (5) 6,5 & (7) 9,1 & (22) 28,6 & (29) 37,7 & (11) 14,3 & (3) 3,9 \\
\hline Tomar minha decisão profissional & (5) 6,5 & (9) 11,7 & (16) 20,8 & (28) 36,4 & (17) 22,1 & (2) 2,6 \\
\hline
\end{tabular}

Em geral, os comentários se referiam às contribuições da intervenção, não restrita à escolha profissional.

Nos questionários destinados aos ex-clientes que interromperam o atendimento havia uma questão sobre os motivos da desistência ou do abandono do processo de orientação profissional. Os dados foram organizados em variáveis específicas do paciente, da instituição, do tratamento e do terapeuta (Lhullier \& Nunes, 2004). $\mathrm{Na}$ análise, verificou-se que os participantes colocam questões de ordem pessoal como os motivos principais para a interrupção do 
atendimento, citando com menor frequência questões de âmbito interpessoal ou problemas relacionados ao serviço, como as questões relativas a limites de horários, número de vagas, condições oferecidas no serviço e competência dos psicólogos estagiários. No entanto, esses pontos destacados podem ser mais bem trabalhados no serviço para o aprimoramento do atendimento oferecido e a formação dos psicólogos estagiários (Arruda, 2009).

Nas sugestões para atendimentos futuros, os participantes (concluintes, abandonadores e desistentes) se referem às atividades e às estratégias utilizadas, notadamente no que se refere a atividades informativas, como palestras, visitas à universidade e debates com alunos e docentes. No serviço, a busca de informação sobre as profissões é estimulada, oferecendo-se revistas, guias e sites da internet para esse fim, mas a preocupação durante o processo de orientação profissional está focada em processar e elaborar tais informações (Spokane, 2004). Assim sendo, considera-se que, mais do que buscar informações, a contribuição do especialista em orientação profissional consiste na adequação do uso das informações no processo de escolha de carreira (Whiston \& Oliver, 2005).

Além dessas sugestões, os participantes incentivam a manutenção do serviço por meio de divulgação e ampliação das vagas oferecidas e tecem elogios acerca da intervenção realizada, o que pode ser entendido como uma valorização do serviço oferecido.

Pode-se inferir, portanto, que os participantes valorizam os resultados da intervenção e veem a realização de atividades exploratórias para obtenção de informação sobre as profissões e suas áreas de atuação como uma estratégia interessante de se aprimorar o serviço. Além disso, a sugestão de ampliação e divulgação pode demonstrar que julgam o trabalho realizado relevante e executado de forma eficaz.

\section{Considerações finais}

De forma geral, os egressos avaliaram positivamente as condições oferecidas pelo serviço, assim como as atividades e os temas trabalhados. Os participantes demonstram satisfação quanto aos resultados da intervenção e ao desenvolvimento de habilidades relativas ao projeto de vida pessoal e profissional. Consideraram o auxílio da orientação profissional importante para o amadurecimento de suas escolhas, porém não the atribuíram papel fundamental, o que é compreensível em razão dos objetivos da intervenção, que foi planejada para que as decisões fossem conscientes e autônomas. Como contribuição ao aprimoramento do serviço, os exclientes sugerem a realização de mais atividades informativas sobre profissões e a divulgação e ampliação de vagas no SOP.

Cumpre destacar alguns limites metodológicos encontrados neste estudo, que podem ser revistos em delineamentos de investigações posteriores. O primeiro refere-se aos intervalos de tempo variáveis entre a intervenção em orientação profissional e a realização da pesquisa. Considera-se que, pelo fato de os ex-clientes estarem em momentos diferentes de vida quando da pesquisa, esse modelo de coleta de dados pode ter influenciado os dados e sugere-se a avaliação em períodos regulares, futuramente. Outra limitação foi o uso do correio como forma de envio dos materiais de coleta de dados. Apesar de ter havido o cuidado de entrar em contato por telefone com os ex-clientes, o número de respostas, ainda que dentro da média descrita na literatura (Selltiz, Wrightsman \& Cook, 1987), foi inferior às expectativas das pesquisadoras. Considera-se que o presente estudo traz reflexões importantes para o aprimoramento do serviço avaliado e que contribuirá para a produção do conhecimento científico na área, sobretudo na questão da avaliação de processos e resultados da intervenção em orientação profissional na realidade brasileira.

\section{Referências}

Almeida, F. H. (2003). Avaliação da intervenção em orientação profissional em uma clínica escola na visão de ex-clientes. (Monografia de Conclusão de curso). Ribeirão Preto, SP: Universidade de São Paulo Programa Optativo de Bacharelado em Psicologia, Faculdade de Filosofia, Ciências e Letras de Ribeirão Preto.

Almeida, F. H. \& Melo-Silva, L. L. (2006). Avaliação de um serviço de orientação profissional: a perspectiva de ex-usuários. Revista Brasileira de Orientação Profissional, 7(2), 81-102.

Amundson, N. E., Harris-Bowlsbey, J. \& Niles, S. G. (2005). Evaluating client progress. Em N. E. Amundson, J. Harris-Bowlsbey \& S. G. Niles (Eds.). Essential elements of career counseling: Process and techniques (pp. 107-117). New Jersey: Pearson Merril Prentice Hall.

Arruda, M. N. F. (2009). Inputs, processos e resultados: Avaliação de um serviço de orientação profissional sob a perspectiva de ex-clientes. (Monografia de Conclusão de curso). Ribeirão Preto, SP: Universidade de 
São Paulo - Faculdade de Filosofia, Ciências e Letras de Ribeirão Preto.

Bardin, L. (1979). Análise de conteúdo. Lisboa, Portugal: Edições 70.

Baudouin, R., Bezanson, L., Borgen, B., Goyer, L., Hiebert, B., Magnusson, K., Michaud, G., Renald, C. \& Turcotte, M. (2007). Demonstrating value: a draft for evaluating the effectiveness of career development interventions. Canadian Journal of Counseling, 41(3), 146-157.

Bohoslavsky, R. (2003). Orientação vocacional: a estratégia clinica (J. M. V. Bojart, Trad.). São Paulo: Martins Fontes.

Brown, D. (2003). Testing and assessment in career development. Em D. Brown (Ed.). Career information, career counseling, and career development (pp. 126-160). Boston: Pearson Education.

Brown, S. D. \& Ryan Krane, N. E. (2000). Four (or five) sessions and a cloud of dust: old assumptions and new observations about career counseling. Em S. D. Brown \& R. W. Lent (Eds.). Handbook of counseling psychology (pp. 740766). Nova Iorque: Wiley \& sons.

Chartrand, J. M. \& Walsh, W. B. (2001). Career assessment: changes and trends. Em F. T. L. Leong \& A. Barak (Eds.). Comtemporary models in vocational psychology: a volume in honor of Samuel $H$. Osipow (pp. 231-253). New Jersey: Lawrence Erlbaum Associates.

Fraga, L. B. (2003). Avaliaşão da intervenção em orientação profissional numa clinica-escola sob a perspectiva do exestagiário. (Monografia de Conclusão de curso). Ribeirão Preto, SP: Universidade de São Paulo Faculdade de Filosofia, Ciências e Letras de Ribeirão Preto.

French, F., Hiebert, B. \& Bezanzon, L. (1994). Introduction: an emerging evaluation model for changing times. Canadian Journal of Counseling, 28(4), 261-269.

Guichard, J. \& Huteau, M. (2001). Avaliação dos efeitos das práticas de orientação. Em Psicologia da orientação (A. P. Silva, Trad., pp. 310-322). Lisboa, Portugal: Instituto Piaget.

Hiebert, B. (1994). A frame work for quality control, accountability and evaluation: being clear about the legitimate outcomes of career counseling. Canadian Journal of Counselling, 28(4), 334-45.

Lassance, M. C. P., Bardag1, M. P. \& I'e1xe1ra, M. A. P. (2009). Avaliação de uma intervenção cognitivo-evolutiva em orientação profissional com um grupo de adolescentes brasileiros. Revista Brasileira de Orientação Profissional, 10 (1), 23-32.

Lhullier, A. C. \& Nunes, M. L. T. (2004). Uma aliança que se rompe. Psicologia: Ciência e Profissão Diálogos, 1, pp. 42-49.

Loosli, L. (2003). Orientação profissional: avaliação do atendimento na visão dos familiares de ex-clientes. (Monografia de Conclusão de curso). Ribeirão Preto, SP: Universidade de São Paulo Faculdade de Filosofia, Ciências e Letras de Ribeirão Preto.

Melo-Silva, L. L. \& Jacquemin, A. (2001). Intervenção em orientação vocacional / profissional: avaliando resultados e processos. São Paulo: Vetor.

Selltiz, C., Wrightsman, L. S. \& Cook, S. W. (1987). Métodos de pesquisa nas relações sociais ( $2^{\mathrm{a}}$ ed.). São Paulo: EPU.

Spokane, A. R. (2004). Avaliação das intervenções de carreira. Em L. M. Leitão (Ed.). Avaliação psicológica em orientação escolar e profissional (pp. 455473). Coimbra: Quarteto.

Swanson, J. L. (1995). The process and outcome of career counseling. Em W. B. Walsh \& S. H. Osipow (Eds.). Handbook of vocational psychology: Theory, research, and practice (pp. 217-259). New Jersey: Lawrence Erlbaum Associates.

Whiston, S. C., Brecheisen, B. K. \& Stephens, J. (2003). Does treatment modality affect career counseling effectiveness? Journal of Vocational Behavior, 62, 390-410.

Whiston, S. C. \& Oliver, L. W. (2005). Career counseling process and outcomes. Em Walsh, W. B. \& Savickas, M. L. (Eds.). Handbook of vocational psychology: theory, research, and practice (pp. 155-194). Londres: Lawrence Erlbaum Associates.

Whiston, S. C. \& Buck, I. M. (2008). Evaluation of career guidance programs. Em J. A. Athanasou \& R. Van Esbroeck (Eds.). International handbook of career guidance (pp. 677-694). London: Springer.

Yokoyama, L. (2009). Orientação profissional em um serviço escola na perspectiva dos familiares de ex-clientes: avaliação 2001-2006. (Monografia de Conclusão de curso). Ribeirão Preto, SP: Universidade de São Paulo - Faculdade de Filosofia, Ciências e Letras de Ribeirão Preto. 
Sobre as autoras:

Marina Noronha Ferraz de Arruda é graduada em Psicologia pela Faculdade de Filosofia, Ciências e Letras de Ribeirão Preto da Universidade de São Paulo (FFCLRP/USP) e pesquisadora em orientação profissional.

Lucy Leal Melo-Silva é psicóloga, docente da Graduação e Pós-Graduação em Psicologia, da Faculdade de Filosofia, Ciências e Letras de Ribeirão Preto (FFCLRP-USP), editora da Revista Brasileira de Orientação Profissional, autora de livros na área da Orientação Profissional e Formação em Psicologia e pesquisadora do CNPq.

Este estudo foi financiado pela Fundação de Amparo à Pesquisa do Estado de São Paulo (FAPESP), por meio de Auxílio Pesquisa destinado a um projeto mais amplo, coordenado pela segunda autora (orientadora) e de Bolsa de Iniciação Científica para a primeira autora, que resultou na monografia disponível no sítio http://portal.ffclrp.usp.br/sites/lucileal/download/454. Outros estudos na mesma linha investigativa estão disponíveis no referido portal. 